\title{
Improving the quality of discharge summaries: implementing updated Academy of Medical Royal Colleges standards at a district general hospital
}

Hannah May-Miller, Joanne Hayter, Lola Loewenthal, Louis Hall, Rebecca Hilbert, Michael Quinn, Nicola Pearson, Alisha Patel, Rebekah Law Epsom and St Helier University Hospitals NHS Trust

\begin{abstract}
Quality of documentation is harder to quantify and incentivise, but it has a significant impact on patient care. Good discharge summaries facilitate continuity between secondary and primary care.

The junior doctors' forum led this project to improve the quality of electronic discharge summaries (eDS). Baseline measurement revealed significant room for improvement. We measured the quality of 10 summaries per month (across all inpatient specialties), against 23 indicators from the revised Academy of Medical Royal Colleges (AoMRC) standards (2013) that were prioritised by GPs as a "minimum dataset".

Junior doctors felt that the Trust's dual eDS systems were responsible for great variation in quality. This was confirmed by the results of a comparison audit of the systems in April 2014: one system greatly outperformed the other (57\% mean compliance with iSoft clinical management (iCM) based system vs. 77\% with InfoPath-based system).
\end{abstract}

We recommended that the Trust move to a single eDS system, decommissioning the iCM-based system, and this proposal was approved by several Trust committees. We worked with information services, junior doctors, general practitioners and hospital physicians to develop and implement a generic template to further improve compliance with AoMRC standards.

In August 2014, the iCM-based system was withdrawn, the new template went live, and training was delivered, coinciding with the changeover of junior doctors to minimise disruption. Median compliance increased from $66.7 \%$ to $77.8 \%$. Quality of discharge summaries had improved across the specialties. There was a reduction in the number of complaints and positive qualitative feedback from general practitioners and junior doctors. Completion of discharge summaries within 24 hours was not affected by this change.

There is still more to be done to improve quality; average compliance with the full AoMRC standards (39 indicators) is $59.5 \%$. With the approval of the Trust executive committee further plan, do, study, act (PDSA) cycles are underway, working to improve the remaining specialtyspecific templates.

\section{Problem}

As the primary method of communication between secondary and primary care, discharge summaries are vital to ensure patient safety and continuity of care. At our Trust, discharge summaries were a common cause of complaint from general practitioners (GPs) and improving the quality of discharge communication was a key priority for the clinical commissioning group, reflected in a locally-set commissioning for quality and innovation (CQUIN) system in 2013/14. Key problem areas were: documentation of investigations, medication changes and follow-up arrangements. This results in poor continuity of patient care from hospital to the community, which jeopardises patient safety.

\section{Background}

In July 2013, the Academy of Medical Royal Colleges (AoMRC) released updated standards for medical record keeping.[1] These included standards for the content of discharge summaries. Clinician variability is hard to affect, but improving systems can have a significant impact, therefore embedding these best practice standards in our technology systems is crucial.

Many quality improvement projects have focused on improving the efficiency of discharge summaries, driven by national commissioning for quality and innovation (CQUIN) targets for electronic discharge summaries to be completed and transmitted to the GP within 24 hours. Fewer projects have focused on the quality of discharge summaries, perhaps as this can be harder to quantify. Several projects have, however, shown changes to electronic systems can improve their quality.[2,3]

Junior doctors at the junior doctors' forum (JDF) led this project. We brainstormed factors that were contributing to poor quality discharge summaries. We hosted a focus group for GP trainees, and emailed all local general practitioners asking for their input. We identified two main areas for improvement: the Trust's electronic discharge summary systems, and training of doctors on how to write discharge summaries. The Trust was using two different electronic discharge summary systems. Both systems had subsections for the doctor to populate with information: the iSoft clinical 
BMJ Quality Improvement Reports

management (iCM) based system had fewer sections, whereas the InfoPath based system offered a more complete and structured template. Discharge summaries could not be drafted and saved using the iCM-based system. This was jeopardising the quality of discharge summaries particularly at weekends and delaying patient discharges.

Anecdotally, the JDF felt that the InfoPath system produced higher quality discharge summaries, but quantitative evidence was needed.

\section{Baseline measurement}

Throughout the year, we sampled 10 discharge summaries per month, across all specialties. We scored each discharge summary against 23 indicators from AoMRC standards that were prioritised by local general practitioners as a "minimum dataset". Baseline measurement revealed great variation in the quality of discharge summaries, and confirmed considerable scope for improvement.

Junior doctors felt that the Trust's dual electronic discharge summary systems were partly responsible for the great variation in quality, therefore we undertook a comparison audit of the systems in April 2014. One hundred and nine discharge summaries across all specialties were measured against the 23 indicators. Results confirmed that one electronic discharge summary system outperformed the other (57\% mean compliance with iCM-based system vs. $77 \%$ with InfoPath-based system).

See supplementary file: ds4331.png - "Graph showing compliance of discharge summaries with the minimum dataset, comparing our two electronic discharge summary systems"

\section{Design}

Initial data showed that the InfoPath-based system outperformed the iCM-based system. The junior doctors' forum met with the medical directors, GP liaison manager and representatives from information services, clinical coding, and clinical audit departments to work through possible solutions and obstacles to change. There was no scope to modify the iCM-based system. All present agreed that the iCM system was not fit for purpose and should be decommissioned. We recognised that this system change may not be easy for those familiar with using the iCM system, so we planned for the system change to take place to coincide with the August changeover of junior doctors to minimise disruption.

The InfoPath based system consists of a number of specialtyspecific templates, but did not have a template for every specialty that would be changing over from the iCM-based system. We designed and tested a new generic template that was fullycompliant with the AoMRC standards, to go live when the iCM system was withdrawn. We sought feedback on the new template from all junior doctors, senior clinicians and local general practitioners. This change was granted formal approval by the clinical information services programme board, operational management group, and Trust executive committee.

\section{Strategy}

PDSA cycle 1: Following discussions at several junior doctors' forum meetings, we concluded that although user-variability played a big part in determining the quality of discharge communication, the electronic systems were not set up to facilitate compliance with AoMRC standards. We also recognised that allowing users to choose between two systems would undermine our quality improvement efforts. We gathered data to support the need for a change.

PDSA cycle 2: After presenting audit data comparing the difference in quality of discharge summaries between the two systems, we applied for and were granted approval by Trust management for the iCM-based discharge summary system to be withdrawn.

PDSA cycle 3: Feedback from junior doctors was that the InfoPath based system was slower to use, and that many of the templates were cumbersome. There was not sufficient time to complete improvements to all 21 specialty-specific templates within the InfoPath system before August, so we worked with information services to develop a generic template that was fully compliant with AoMRC guidance. We emailed all local general practitioners and asked for their feedback on this draft template.

PDSA cycle 4: Following GP feedback, we incorporated several sections into the new template, to remind doctors to provide advice about fitness for work and safe discharge of patients on warfarin. We finalised the user interface of the template, and invited several junior doctors to test the template for real discharge summaries. Feedback was positive and no further changes were made. Information services completed the build of the template.

PDSA cycle 5: On 6th August, the iCM-based system was withdrawn and the generic template went live. Training was arranged for new starters on "How to write a good discharge summary", incorporating many real-life frustrations that had been raised by GPs. Re-audit of the quality of discharge summaries took place showing significant progress had been made. Analysis of the re-audit data shows that the new generic template can be improved further; some sections are still poorly completed. There is significant variability between the other specialty-specific templates, many of which lack key sections.

\section{Results}

Little improvement in the quality of discharge summaries was seen during the consultation and design cycles of the project (PDSA cycles 1 to 4), but median compliance increased from $66.7 \%$ to $77.8 \%$ after the system change in August 2014. Comparison of the April and September audit data showed that average compliance with the minimum dataset ( 23 indicators) improved from $67.9 \%$ to $75.7 \%$ ( $P<0.001$ with an unpaired T-test). Compliance has improved across the specialties, except for renal medicine who never used the iCM-based system. From September onward, we additionally measured discharge summaries against the full AoMRC standards (39 indicators) and found that there is significant room for 
improvement; average compliance in September was 59.5\%. The new generic template performs better than the other templates (67.4\% vs. $59.6 \%, \mathrm{P}<0.0001)$.

There had been concern that the template-based system would increase the time taken to complete a discharge summary and reduce the percentage of summaries completed within 24 hours of a patient's discharge, however analysis of Trust data shows that this has not been the case. Verbal feedback from junior doctors has also been positive. We have identified a few system glitches that have been fed back to information services.

We have also seen a decrease in the number of complaints from general practitioners by the GP liaison office relating to the quality of discharge summaries (May to July 2014: four complaints received, August to October 2014: one complaint received) and have received a number of personal messages of thanks from general practitioners.

See supplementary file: ds5198.png - "Run chart of the quality of discharge summaries, 2014."

\section{Lessons and limitations}

To bring about this significant change to fundamental systems, the junior doctors' forum was invaluable as a platform through which junior doctors can collaborate and lead an improvement initiative. This project was a success because the problem was a priority for junior doctors, GPs, and the Trust. To engage others in a largescale project, it is worth identifying different motivations. Involving information services from the beginning avoided wasting time as it ensured that the proposed intervention was achievable. Although changes to information services systems are hard to achieve, they lead to sustained improvements.

One limitation when interpreting the data is that with a changeover of junior doctors in August, the improvement could be due to a more conscientious cohort of new foundation year one doctors completing the summaries. Although it is possible that doctors become more complacent the longer they have been at the Trust, some sections such as allergies were not present on the iCM system and so even the most conscientious doctor would not remember to include this information. Month to month data suggests that the change has eliminated the poorest quality discharge summaries.

\section{Conclusion}

Moving to one electronic discharge summary system and designing a new template has led to a significant improvement in the quality of discharge summaries at Epsom and St Helier University Hospitals NHS Trust. This improves patient care and safety. The re-audit has identified some areas that could be further improved. With a unified electronic system, we are continuing to work with information services to improve the specialty-specific templates. Clinician variability is hard to affect, but improving systems can have a significant impact. Embedding best practice standards in the design of our electronic discharge summary system has been crucial to the success of this intervention.

Following its successful first phase, the junior doctors' forum has been granted approval by the Trust executive committee to continue to work with information services on this project. Work is underway to revise each of the specialty-specific templates, improving uniformity, and incorporate key missing sections such as "allergies". Data collection is ongoing, with specialty templates being revised and introduced in turn.

\section{References}

1. Health and Social Care Information Centre, Academy of Medical Royal Colleges. Standards for the clinical structure and content of patient records. London: HSCIC, 2013. http://www.rcplondon.ac.uk/resources/standards-clinicalstructure-and-content-patient-records (accessed 9th June 2014).

2. O'Leary KJ, Liebovitz DM, Feingladd J, Liss DT, Evans DB, Kulkami N, Landler MP, Baker DW. Creating a better discharge summary: improvement in quality and timeliness using an electronic discharge summary. J Hosp Med 2009;4(4):219-25.

3. Maurice AP, Chan S, Pollard CW, Kidd RA, Ayre SJ, Ward HE, Walter DL. Improving the quality of hospital discharge summaries utilising an electronic prompting system. BMJ Qual Improv Report 2014;3:1.

\section{Declaration of interests}

Nothing to declare.

\section{Acknowledgements}

Lola Loewenthal, Joanna Sutherby, Abbey Cargill, Sarah Kennie, Barry Mullins, Nicola Pearson, Joshua Wolrich, Joyce Rochester, Sathya Sekar, Catherine Townend, lan Barnard, Janet Samuel, Alison Richmond, James Marsh, Ruth Charlton.

\section{Ethical approval}

Ethics approval not sought; this project involved systematic review of discharge communication, a standard aspect of patient care, against nationally agreed quality criteria,[1] with the aim of improvement in patient care. 